\title{
Enseñar, innovar y reflexionar en la Asignatura Adolescencia y Riesgo
}

\section{Teach, innovate and reflect on the Subject Adolescence and Risk}

LORENA TARRIÑO CONCEJERO

ORCID: https://orcid.org/0000-0003-0158-5744

Universidad de Sevilla.

Departamento de Enfermería.

Itarrino@us.es

Fecha de recepción: 14-11-2019

Fecha de aceptación: 19-11-2019

DOI: http://dx.doi.org/10.12795/9788447221912.035

Pp.: 801-818 


\section{Resumen}

Las nuevas realidades que están presentes en nuestras aulas hacen necesaria una reformulación de nuestra práctica docente. Para ello, es necesario implantar de manera gradual mejoras e innovaciones en los planes de estudios. Se presenta un ciclo de mejora docente en la asignatura Adolescencia y Riesgo del Grado de Enfermería. Se utilizaron diferentes metodologías, como lectura crítica de artículos científicos, videoforums, roles playing, realización de informes y gamificación. La evaluación del proceso resultó satisfactoria y con un alto porcentaje de implicación del alumnado.

Palabras claves: Adolescencia y riesgo; Grado enfermería; Docencia Universitaria; Experimentación Docente Universitaria; Innovación.

\section{Abstract}

The new realities that we currently have in our classrooms, necessitates a reformulation of our teaching practice. For this, it is necessary to gradually implement improvements and innovations in the study program.A cycle of teacher improvement in the subject Adolescence and Risk of Nursing Degree is presented. Different methodologies were used such as critical reading of scientific articles, videoforums, playing roles, reporting and gamification.The evaluation of the process was satisfactory and with a high percentage of student involvement.

Key words: Adolescence and risk; Nursing degree; UniversityTteaching; University Teaching Experimentation; Innovation.

Jornadas de Formación e Innovación Docente del Profesorado | № 2 (2019)

Esta obra se distribuye con la licencia Creative Commons 


\section{Descripción del contexto de intervención}

La mejora docente, o ciclo de mejora docente (CMD), que se presenta se realiza en 40 curso del Grado en Enfermería, en una asignatura optativa de 6 créditos ECTS, Adolescencia y Riesgo. Las sesiones son de 4 horas, los martes de 16 a 20 h. en el primer cuatrimestre. El grupo lo forman 7 alumnas, que deciden elegir esta optativa por estar interesadas en la etapa adolescente y las conductas de riesgo que se muestran en la misma.

El CMD se ha aplicado en 8 horas dos martes, en los cuales se ha trabajado contenido conceptual, procedimental y actitudinal relacionado con la Violencia de género en la adolescencia -con una duración de 4 horas- y sexualidad, embarazos no deseados y enfermedades de transmisión sexual -las 4 horas restantes-.

En esta asignatura se realizó anteriormente un primer ciclo de mejora de 4 horas, que sirvió para preparar el presente y considerar más al alumnado en todas sus fases. Para el desarrollo de ambos ciclos he tenido en cuenta las aportaciones y experiencia vivida en el Curso General de Docencia Universitaria de la Universidad de Sevilla y las diferentes lecturas de Porlán (2017), Bein (2007) y Finkel (2008).

\section{Diseño del CMD}

\subsection{Preguntas claves o problemas de interés y mapas de contenidos}

Los diferentes temas se han abordado en las dos sesiones con preguntas claves o problemas de interés, que han enlazado los diferentes tipos de contenidos: conceptuales, procedimentales y actitudinales.

Jornadas de Formación e Innovación Docente del Profesorado | № 2 (2019)

Esta obra se distribuye con la licencia Creative Commons 
En la figura 1 se expone el mapa de contenidos relacionado con la violencia de género en la adolescencia (4 horas) y en la figura 2 y 3 los mapas de contenidos de sexualidad, embarazo no deseado e infecciones de transmisión sexual (4h).

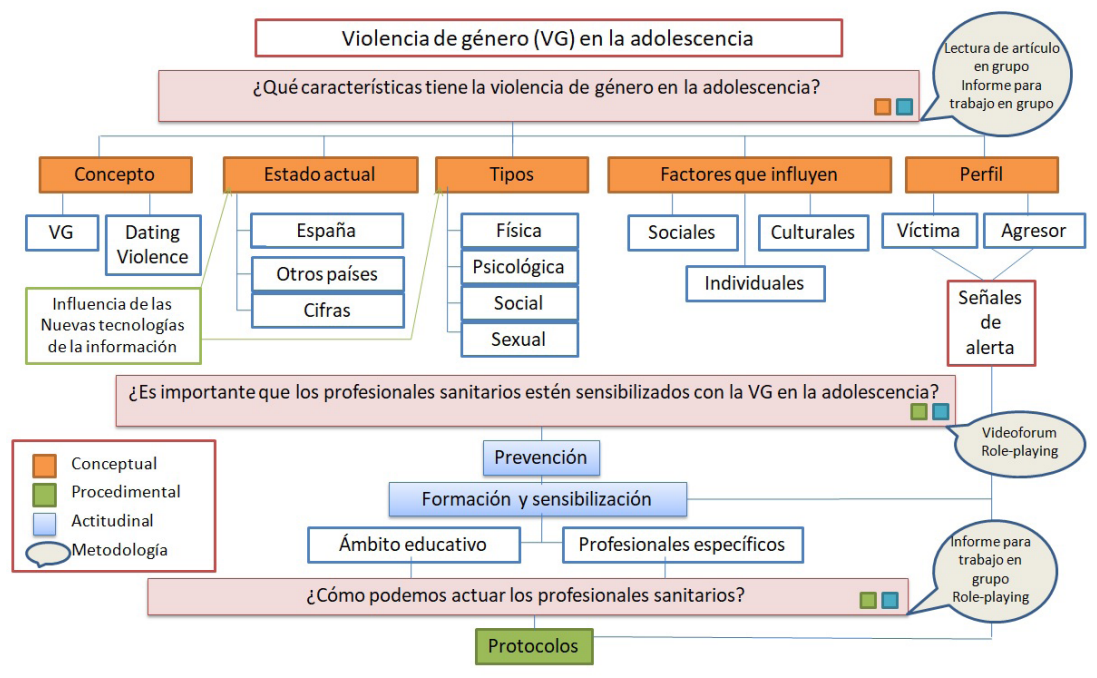

Figura 1. Mapa conceptual: Violencia de género (VG) en la adolescencia

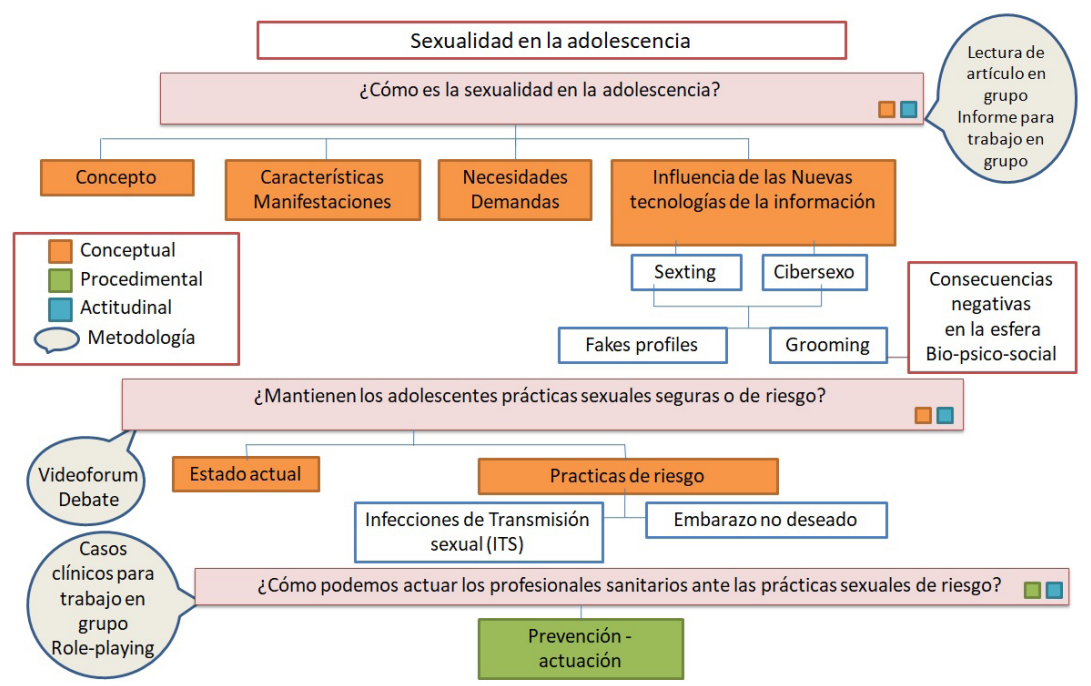

Figura 2. Mapa conceptual: Sexualidad en la adolescencia (1)

Jornadas de Formación e Innovación Docente del Profesorado | № 2 (2019)

Esta obra se distribuye con la licencia Creative Commons Reconocimiento-NoComercial-SinObraDerivada $\quad 4.0$ Internacional (CC BY-NC-ND 4.0.) 


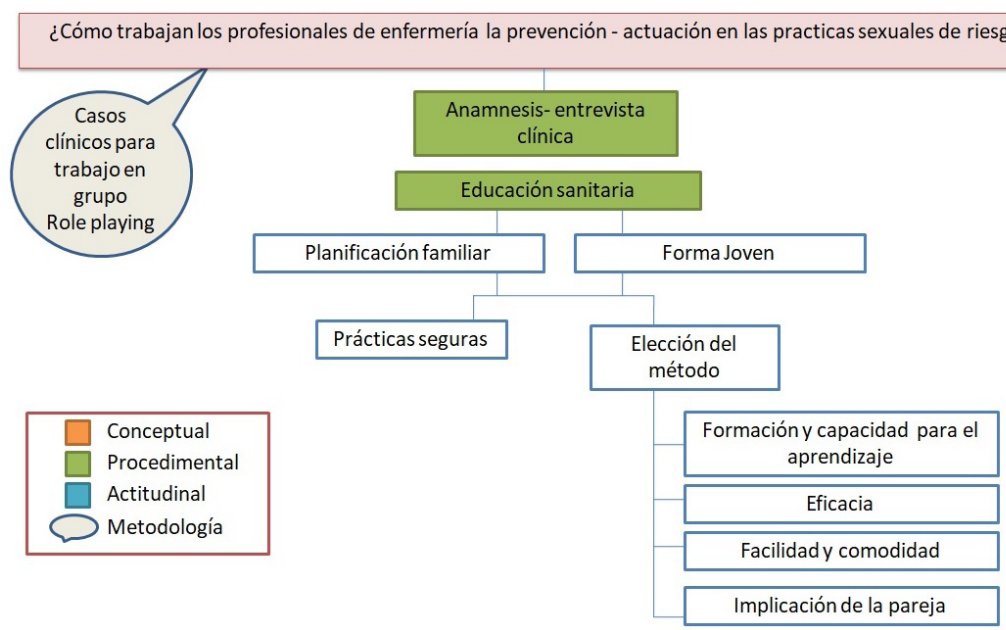

Figura 3. Mapa conceptual: Sexualidad en la adolescencia (2)

\section{Modelo metodológico y secuencia de actividades}

El modelo metodológico que se presenta a continuación (Figura 4) consta de 7 fases en un sentido unidireccional, pero que se retroalimentan unas de otras.

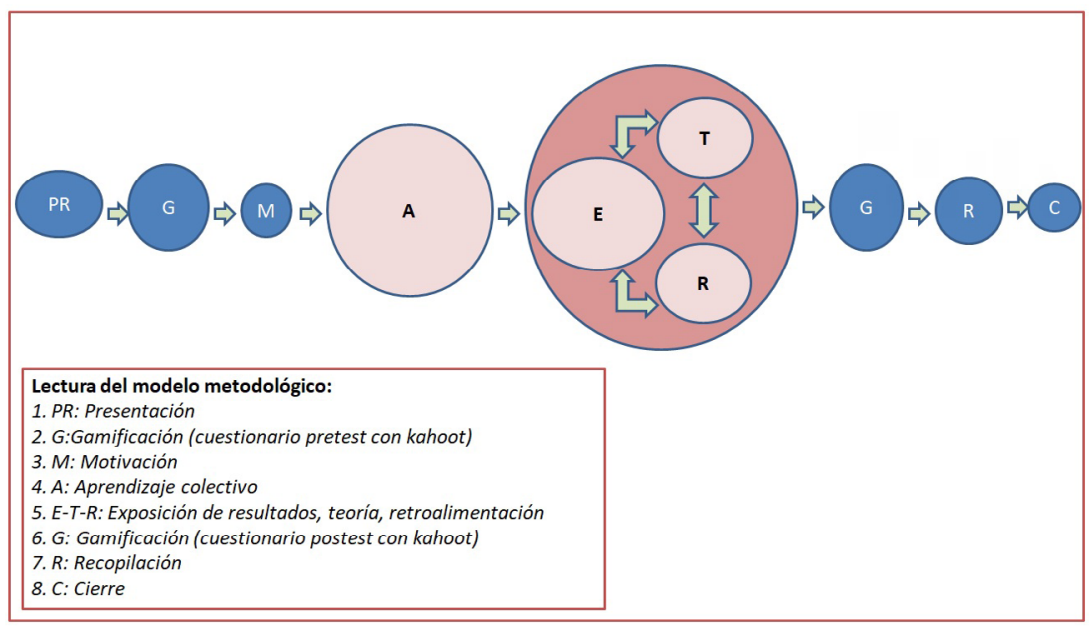

Figura 4: Modelo metodológico aplicado en el aula

La secuencia de actividades, tiempo empleado, recursos y tipos de contenidos trabajados (conceptual,

Jornadas de Formación e Innovación Docente del Profesorado | № 2 (2019)

Esta obra se distribuye con la licencia Creative Commons Reconocimiento-NoComercial-SinObraDerivada Internacional (CC BY-NC-ND 4.0.) 
procedimental o actitudinal) en cada una de las sesiones, se expone en la siguiente tabla (Tabla1).

\section{Tabla 1: Secuencia de actividades}

\begin{tabular}{|c|c|c|}
\hline \multicolumn{3}{|l|}{ Fase 1. PR: Presentación } \\
\hline \multicolumn{3}{|l|}{ Actividades } \\
\hline \multicolumn{3}{|c|}{ Presentación en PowerPoint de los contenidos a trabajar. } \\
\hline Recursos & Tiempo & Contenidos \\
\hline $\begin{array}{l}\text { Ordenador y presentación } \\
\text { PowerPoint }\end{array}$ & 15 minutos (por sesión) & Conceptual \\
\hline \multicolumn{3}{|l|}{ Fase 2. G: Gamificación } \\
\hline \multicolumn{3}{|l|}{ Actividades } \\
\hline \multicolumn{3}{|c|}{$\begin{array}{l}\text { Sesión 1: Cuestionario (en Kahoot, 1a sesión y en Google forms, 20 sesión) para } \\
\text { evaluar el conocimiento previo de la materia (las preguntas del mismo se exponen } \\
\text { en evaluación). }\end{array}$} \\
\hline Recursos & Tiempo & Contenidos \\
\hline $\begin{array}{l}\text { Ordenador profesor con } \\
\text { exposición de preguntas } \\
\text { Móviles para contestar }\end{array}$ & 15 minutos (por sesión) & $\begin{array}{l}\text { Conceptual } \\
\text { Actitudinal } \\
\text { Procedimental }\end{array}$ \\
\hline \multicolumn{3}{|l|}{ Fase 3. Motivación } \\
\hline \multicolumn{3}{|l|}{ Actividades } \\
\hline \multicolumn{3}{|c|}{$\begin{array}{l}\text { Sesión 1: Frase motivadora: La disciplina es el puente entre metas y logros. Autor Jim } \\
\text { Rohn .. } \\
\text { Sesión 2: Frase motivadora: Cree en ti mismo y en lo que eres. Se consciente de que } \\
\text { hay algo en tu interior que es más grande que cualquier obstáculo. Autor: Christian } \\
\text { D. Larson. } \\
\text { Objetivo es que el alumnado reflexione sobre el significado de la frase, y motivar al } \\
\text { alumnado. }\end{array}$} \\
\hline Recursos & Tiempo & Contenidos \\
\hline $\begin{array}{l}\text { Ordenador } \\
\text { Presentación PowerPoint } \\
\text { con frase e imagen. }\end{array}$ & 5 minutos (por sesión) & Actitudinal \\
\hline \multicolumn{3}{|l|}{ Fase 4. Aprendizaje Colectivo } \\
\hline \multicolumn{3}{|l|}{ Actividades } \\
\hline $\begin{array}{l}\text { Según la pregunta de intere } \\
\text { actividades. }\end{array}$ & el alumnado tendrá qu & bajar en grupo diferentes \\
\hline
\end{tabular}

Jornadas de Formación e Innovación Docente del Profesorado | № 2 (2019)

Esta obra se distribuye con la licencia Creative Commons Reconocimiento-NoComercial-SinObraDerivada $\quad 4.0$ Internacional (CC BY-NC-ND 4.0.) 
Sesión 1

Pregunta 1: ¿Qué características tiene la violencia de género en la adolescencia?

- Se trabajará sobre Lectura de artículo en grupo e Informe (con sus objetivos) para

trabajo en grupo.

- Deberán reflejar todo el contenido que se ha marcado en el Informe a través

de una búsqueda en bases de datos, en la lectura del artículo en grupo y de los

artículos facilitados en enseñanza virtual.

Pregunta 2: ¿Es importante que los profesionales sanitarios estén sensibilizados con

la VG en la adolescencia?

- Se expondrán varios videos relacionados con la materia, se suscitará un debate, y deberán trabajar el contenido expuesto mediante Role-playing.

Pregunta 3: ¿Cómo podemos actuar los profesionales sanitarios?

- Mediante el documento del Protocolo de actuación subido a Enseñanza virtual, deberán realizar un pequeño informe de puntos clave y una representación mediante Role-playing.

Sesión 2

Pregunta 1: ¿Cómo es la sexualidad en la adolescencia?

- Se trabajará sobre Lectura de artículo en grupo e Informe (con sus objetivos) para trabajo en grupo.

- Deberán reflejar todo el contenido que se ha marcado en el Informe a través de una lectura de un artículo en grupo y de varios artículos facilitados en enseñanza virtual.

Pregunta 2: ¿Mantienen los adolescentes prácticas sexuales seguras o de riesgo?

- Se expondrán varios videos relacionados con la materia, se suscitará un debate, y

deberán trabajar el contenido expuesto mediante Role playing.

Pregunta 3 y 4: ¿Cómo podemos actuar los profesionales sanitarios ante las

prácticas sexuales de riesgo?; ¿Cómo trabajan los profesionales de enfermería la prevención - actuación en las prácticas sexuales de riesgos.

- Mediante 3 casos clínicos que versan sobre diferentes prácticas de riesgos y sus consecuencias, el alumnado deberá resolver los objetivos de cada caso, dándoles una representación mediante Role playing.

\begin{tabular}{|c|c|c|c|}
\hline Recursos & \multicolumn{2}{|l|}{ Tiempo } & Contenidos \\
\hline \multirow[b]{2}{*}{$\begin{array}{l}\text { Portátiles } \\
\text { Acceso a internet } \\
\text { Guión o índices para } \\
\text { realizar los informes. } \\
\text { Artículos o documentos } \\
\text { para realizar el } \\
\text { Role-playing } \\
\text { Distintos materiales para } \\
\text { ejecutar el Role-playing }\end{array}$} & Sesión 1 & Sesión 2 & \multirow[b]{2}{*}{$\begin{array}{l}\text { Pregunta 1: Conceptuales y } \\
\text { actitudinales } \\
\text { Pregunta } 2 \text { : Procedimental } \\
\text { y actitudinal } \\
\text { Pregunta } 3 \text { : Procedimental } \\
\text { y actitudinal* }\end{array}$} \\
\hline & $\begin{array}{l}1 \text { hora y } 30 \\
\text { minutos. } \\
\text { Pregunta1: } \\
40 \text { min. } \\
\text { Pregunta2: } \\
20 \text { min. } \\
\text { Pregunta3: } \\
30 \text { min }\end{array}$ & $\begin{array}{l}1 \text { hora y } 30 \\
\text { minutos. } \\
\text { Pregunta1: } \\
30 \text { min. } \\
\text { Pregunta2: } \\
20 \text { min. } \\
\text { Pregunta3: } \\
40 \text { min. }\end{array}$ & \\
\hline
\end{tabular}

Jornadas de Formación e Innovación Docente del Profesorado | № 2 (2019)

Esta obra se distribuye con la licencia Creative Commons Reconocimiento-NoComercial-SinObraDerivada $\quad 4.0$ Internacional (CC BY-NC-ND 4.0.) 
*Los contenidos actitudinales se trabajaran en todo el todas las preguntas de interés, porque se evaluará la tener una actitud crítica en la búsqueda y lectura de artículos, relación en el trabajo en grupo, tener una actitud activa y comprensiva, entrega de informes con rigor.

También se evaluará escuchar y prestar atención a los videos expuestos; y realizar con una actitud profesional y comprometida los distintos Role-playing.

Fase 5. E-T-R: Exposición de resultados, teoría, retroalimentación Actividades

El alumnado expondrá los resultados ya sea en forma de informe, resolución de casos, o por Role-playing, al mismo tiempo se irá entrelazando con la teoría indispensable que deben conocer, se reforzará los conocimientos adecuados y se corregirán los equívocos, mostrándoles el adecuado. Todo ello con una retroalimentación continua, activa y positiva favoreciendo un debate constructivo en el aula.

\begin{tabular}{|l|l|l|}
\hline Recursos & Tiempo & Contenidos \\
\hline Informes & El desarrollo de las tres & Conceptuales, actitudinales \\
Casos clínicos & $\begin{array}{l}\text { preguntas debe de ser en } \\
\text { 1hora y 20 minutos (por }\end{array}$ & y procedimentales. \\
$\begin{array}{l}\text { Materiales diversos } \\
\text { para escenificar el } \\
\text { Role-playing. }\end{array}$ & sesión). & \\
\hline
\end{tabular}

Fase 6. G: Gamificación

Actividades

Cuestionario (en Kahoot, sesión 1ạ y el Google forms, 2o sesión) para evaluar el conocimiento después de trabajar la materia (las preguntas del mismo se exponen en evaluación).

\begin{tabular}{|l|l|l|}
\hline Recursos & Tiempo & Contenidos \\
\hline $\begin{array}{l}\text { Ordenador profesor con } \\
\text { exposición de preguntas } \\
\text { Móviles para contestar }\end{array}$ & 15 minutos (por sesión). & $\begin{array}{l}\text { Conceptual } \\
\text { Actitudinal } \\
\text { Procedimental }\end{array}$ \\
\hline Fase 7. R: Recopilación & \multicolumn{2}{|l|}{} \\
\hline Actividades & Tiempo & Contenidos \\
\hline Síntesis y resumen de lo más importante de la materiaa & Conceptual \\
\hline Recursos & 15 minutos (por sesión). \\
\hline $\begin{array}{l}\text { PowerPoint con } \\
\text { presentación }\end{array}$ & \multicolumn{2}{|l}{} \\
\hline
\end{tabular}

Jornadas de Formación e Innovación Docente del Profesorado I № 2 (2019)

Esta obra se distribuye con la licencia Creative Commons Reconocimiento-NoComercial-SinObraDerivada $\quad 4.0$ Internacional (CC BY-NC-ND 4.0.) 
Cerrar la sesión planteando siempre la importancia de la materia trabajada sobre la profesión.

Sesión 1: ¿Es importante conocer y abordar de manera efectiva la VG en la adolescencia en Enfermería?

Sesión 2: ¿Es importante el papel que tiene el profesional de enfermería en la prevención y actuación en las prácticas de riesgo?

\begin{tabular}{|l|l|l|}
\hline Recursos & Tiempo & Contenidos \\
\hline $\begin{array}{l}\text { Planteamiento de la } \\
\text { pregunta }\end{array}$ & 5 minutos (por sesión). & $\begin{array}{l}\text { Conceptual } \\
\text { Actitudinal } \\
\text { Procedimental }\end{array}$ \\
\hline
\end{tabular}

\section{Cuestionario inicial-final para evaluar el aprendizaje de los estudiantes}

Se diseñó un cuestionario inicial con el objetivo de analizar el punto de partida del alumnado y el grado de conocimiento sobre los contenidos a impartir y un cuestionario final para determinar el grado de aprendizaje adquirido por el alumnado. Las preguntas fueron las mismas pero con un orden distinto.

Dichos cuestionarios se elaboraron mediante Kahoot (con 4 opciones de respuesta y sólo una correcta) en la primera sesión y mediante Google forms (con preguntas abiertas) en la segunda sesión (Tabla 2).

Tabla 2. Preguntas de los cuestionarios inicial y final

Preguntas de la 1o sesión relacionadas con la Violencia de género en la adolescencia

¿Qué es la violencia de género?

¿Qué tipo de violencia de género existe en el noviazgo?

¿Qué factores influyen en la violencia de género?

Los profesionales sanitarios deben....

¿Cómo se denomina la secuencia de actuación que debe seguir un enfermero ante una víctima de VG?

¿Para qué sirve esa secuencia de actuación?

Preguntas de la 2 o sesión relacionadas con la Sexualidad en la adolescencia

Jornadas de Formación e Innovación Docente del Profesorado | № 2 (2019)

Esta obra se distribuye con la licencia Creative Commons Reconocimiento-NoComercial-SinObraDerivada $\quad 4.0$ Internacional (CC BY-NC-ND 4.0.) 


\section{Aplicación del CMD}

\section{Desarrollo real de las sesiones. Análisis y valoración}

Para analizar lo que ocurría en mis clases utilicé el diario del profesor, propuesto por Porlán y Martín (1991). En él anoté todo lo que ocurría en el aula, para poder analizar y extraer los aspectos más relevantes para mejorar mi docencia.

El desarrollo real de las dos sesiones ha sido el mismo que el propuesto, se ha aplicado el modelo metodológico posible diseñado para este ciclo de 8 horas sin dificultad, siguiendo las distintas fases del mismo.

La temporalización no se ha cumplido para totas las actividades diseñadas, algunas se han desarrollado con más lentitud (fase 4 y 5) y otras, sin embargo, han terminado antes (fase 7). En la segunda sesión se introdujeron dos actividades más; la primera fue una actividad de gamificación con Mentimeter (Imagen 1) entre la 3 y 4 fase. Esta gamificación no la habían utilizado antes y fue muy sugerente para las alumnas. La segunda, fue un análisis breve de un artículo publicado por 20 minutos ese mismo día, sobre las relaciones de riesgo en adolescentes (entre la fase 4 y 5). Ambas actividades propiciaron un debate muy nutritivo y activó a las alumnas para las siguientes actividades.

Jornadas de Formación e Innovación Docente del Profesorado | № 2 (2019) 


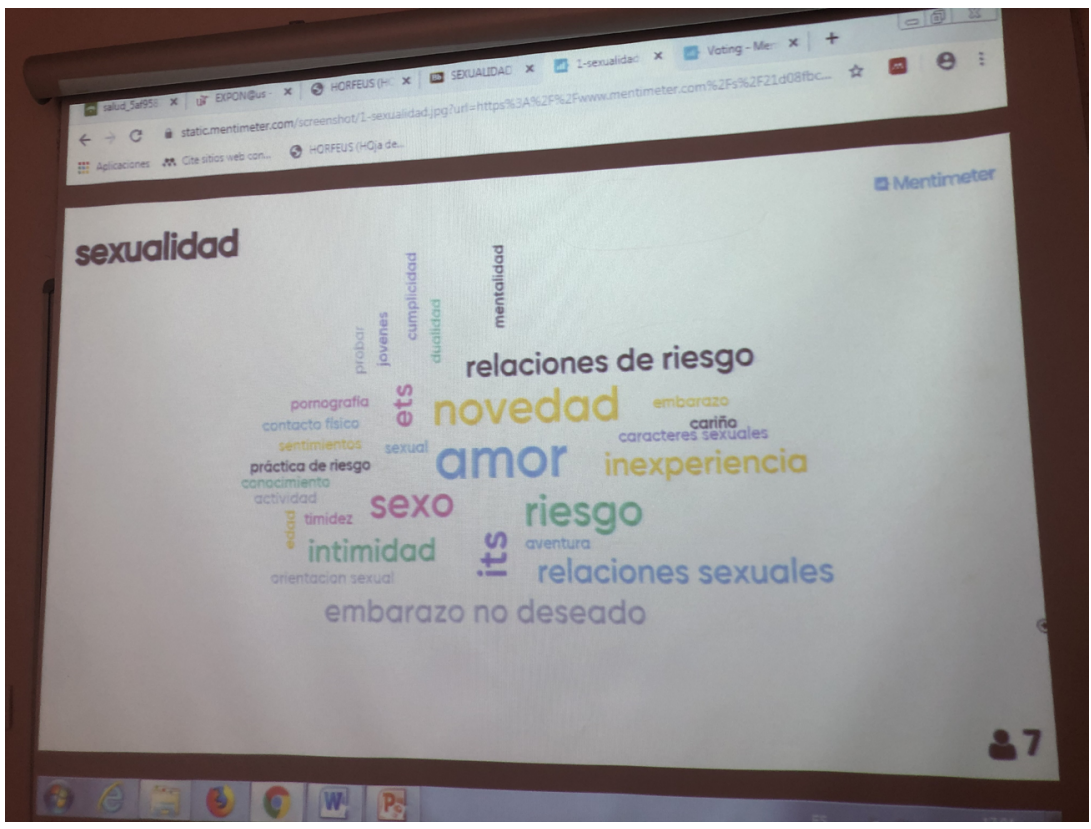

Imagen 1. Fotografía de Mentimeter en el aula

Lo que más impactó en las alumnas fue el uso de las nuevas tecnologías de la información, el Kahoot, y los videoforums, por eso decidí introducir una gamificación (Mentimeter) más en la 2a sesión. La exposición de los videos suscitó un debate abierto muy enriquecedor, que se completó en un grado mayor (que yo no esperaba) con los Roles playing, pues sus actitudes fueron un reflejo fiel de lo que yo realmente quería trabajar a nivel actitudinal, procedimental y conceptual. La utilización de casos clínicos y su posterior role playing las aproximó a la realidad exterior, gustándole mucho la experiencia. Por el contrario, lo que menos les motivó fue la lectura crítica pues, según me transmitieron, el lenguaje de los diferentes artículos no estaba adecuado a su nivel de competencias.

En resumen, el grupo de alumnas ha tenido una magnífica participación en cada una de las actividades, resultando en un aprendizaje colaborativo exitoso. La mayoría de las tareas han sido desarrolladas con aplicación a la

Jornadas de Formación e Innovación Docente del Profesorado | № 2 (2019)

Esta obra se distribuye con la licencia Creative Commons 
práctica, lo que ha motivado mucho al grupo, y con los debates y los roles playing se ha enriquecido mucho sus actitudes ante posibles situaciones futuras en la práctica clínica.

Por último, en relación a la valoración del CMD, me gustaría transmitir mis emociones al respecto:

- Me he encontrado mucho más confiada que en el primer ciclo de mejora, pues ya tenía un proyecto piloto validado y conocía con más profundidad a las alumnas y la dinámica de la clase.

- Mi mayor miedo sigue siendo que la utilización de videoforum y los roles playing propician debates o interpretaciones muy abiertas, y me preocupa que a lo mejor me pregunten algo que no sepa cómo abordarlo. También que, en ocasiones, los temas de conversación se pueden dirigir hacia otros asuntos que no estén contemplados en esa sesión, pero que en realidad están relacionados, y no sé cómo lo abordaría o reconduciría al tema central de nuestro interés.

\section{Evaluación del aprendizaje de las estudiantes}

Las respuestas de los cuestionarios fueron analizadas detectando el escalón de aprendizaje en el que cada una se situaba. Esto permite reflexionar sobre qué aspectos de nuestra docencia o de sus modelos mentales presentan un posible bloqueo de adquisición para pasar a un nivel más elevado de la escalera.

Toda esta información se representó en lo que denominamos escaleras de aprendizaje, en las cuales se muestra el nivel de aprendizaje, los obstáculos entre un nivel y el siguiente y el porcentaje inicial y final del alumnado que se situó en cada escalón. Conforme asciendes en la escalera, el nivel de aprendizaje es mayor (aumenta el grado de complejidad de la respuesta), superándose los obstáculos

Jornadas de Formación e Innovación Docente del Profesorado | № 2 (2019)

Esta obra se distribuye con la licencia Creative Commons 
que antes no permitían adquirir el nivel de aprendizaje superior. A continuación se representan, a modo de ejemplo, dos escaleras de aprendizaje, una por cada sesión (Figura 5 y 6$)$.

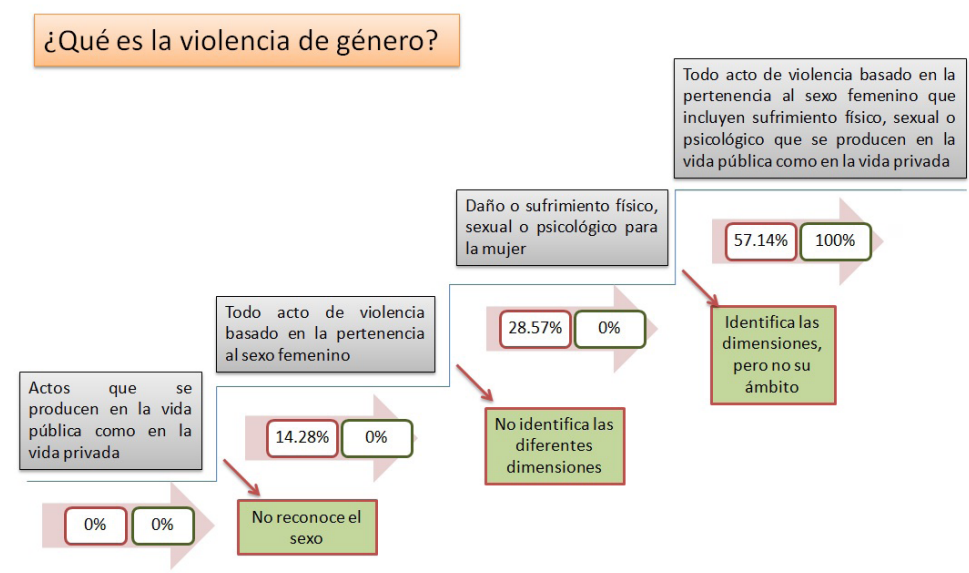

Figura 5. Escalera de aprendizaje para la pregunta 1 (Sesión 1)

¿Qué es una práctica de riesgo relacionada con la sexualidad?

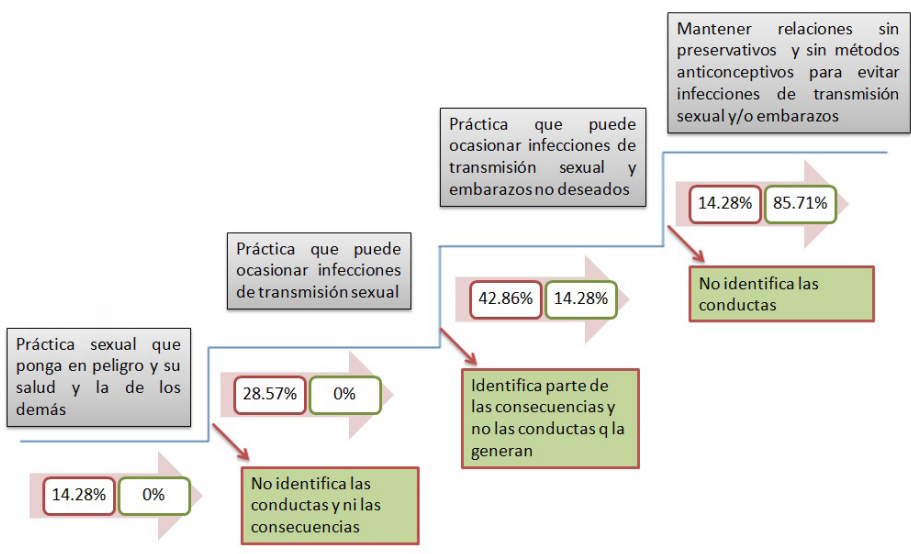

Figura 6. Escalera de aprendizaje para la pregunta 3 (Sesión 2)

En el análisis de todas las preguntas se observa una evolución hacia un mayor nivel de complejidad en sus modelos mentales, lo que indica que las estudiantes pudieron superar los obstáculos detectados al inicio de la

Jornadas de Formación e Innovación Docente del Profesorado | № 2 (2019)

Esta obra se distribuye con la licencia Creative Commons 
sesión. En ninguna de ellas hubo retroceso. Esto puede justificarse por el escaso número de alumnas, que propició un buen ambiente en el aula, y porque determinados contenidos ya se habían mostrados en otras asignaturas del grado en años anteriores.

A continuación se expone la tabla 2, donde se puede observar el nivel de aprendizaje de cada alumna en el cuestionario inicial y final de la sesión 1.

Tabla 2. Evolución en el nivel de aprendizaje de cada alumna

\begin{tabular}{|c|c|c|c|c|c|c|c|c|c|c|c|c|c|c|c|c|c|c|}
\hline \multirow{2}{*}{$\begin{array}{l}\text { Alumnas } \\
\text { Alum. } 1\end{array}$} & \multicolumn{3}{|c|}{$\begin{array}{l}\text { Pregunta } \\
1\end{array}$} & \multicolumn{3}{|c|}{$\begin{array}{l}\text { Pregunta } \\
2\end{array}$} & \multicolumn{3}{|c|}{$\begin{array}{l}\text { Pregunta } \\
3\end{array}$} & \multicolumn{3}{|c|}{$\begin{array}{l}\text { Pregunta } \\
4\end{array}$} & \multicolumn{3}{|c|}{$\begin{array}{l}\text { Pregunta } \\
5\end{array}$} & \multicolumn{3}{|c|}{$\begin{array}{l}\text { Pregunta } \\
6\end{array}$} \\
\hline & B & $\mathrm{D}$ & $\uparrow$ & A & $\mathrm{C}$ & $=$ & $\mathrm{C}$ & $\mathrm{D}$ & $\uparrow$ & B & A & $\uparrow$ & B & $\mathrm{C}$ & $\uparrow$ & $\mathrm{D}$ & $\mathrm{C}$ & $\uparrow$ \\
\hline Alum. 2 & $\mathrm{C}$ & $\mathrm{B}$ & $\rightarrow$ & A & D & $\uparrow$ & $\mathrm{C}$ & D & $\uparrow \uparrow$ & B & A & $\uparrow$ & B & $\mathrm{C}$ & $\uparrow$ & $\mathrm{B}$ & $\mathrm{C}$ & $\uparrow$ \\
\hline Alum. 3 & B & D & $\uparrow$ & A & D & $\uparrow$ & $\mathrm{A}$ & A & $=$ & B & $\mathrm{C}$ & $\rightarrow$ & B & $\mathrm{C}$ & $\uparrow$ & $\mathrm{A}$ & D & $\rightarrow$ \\
\hline 1.4 & B & $\mathrm{D}$ & $\uparrow$ & B & $\mathrm{D}$ & $\uparrow$ & $\mathrm{C}$ & $\mathrm{D}$ & 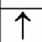 & B & $\mathrm{A}$ & $\uparrow$ & $\mathrm{D}$ & $\mathrm{C}$ & $\uparrow$ & $\mathrm{D}$ & $\mathrm{C}$ & $\uparrow$ \\
\hline Alum. 5 & B & D & $\uparrow$ & A & $\mathrm{A}$ & $=$ & B & $\mathrm{D}$ & $\uparrow$ & $\mathrm{C}$ & $\mathrm{A}$ & $\uparrow$ & B & $\mathrm{C}$ & $\uparrow$ & $\mathrm{D}$ & $\mathrm{C}$ & $\uparrow$ \\
\hline Alum. 6 & $\mathrm{C}$ & B & $\rightarrow$ & $\mathrm{C}$ & A & $\rightarrow$ & B & $\mathrm{C}$ & $\uparrow$ & B & $\mathrm{C}$ & $\rightarrow$ & $\mathrm{D}$ & $\mathrm{C}$ & $\uparrow$ & B & $\mathrm{D}$ & $\rightarrow$ \\
\hline Alum. 7 & B & A & $\rightarrow$ & B & D & $\uparrow$ & $\mathrm{C}$ & $\mathrm{D}$ & $\uparrow$ & $\mathrm{C}$ & $\mathrm{A}$ & $\uparrow$ & D & $\mathrm{C}$ & $\uparrow$ & $\mathrm{A}$ & D & $\rightarrow$ \\
\hline \multicolumn{19}{|c|}{ ectura de la tabla } \\
\hline \multicolumn{8}{|c|}{$\begin{array}{l}\leftarrow \text { retrocede de nivel } \\
=\text { Se queda en el mismo nivel. }\end{array}$} & \multicolumn{11}{|c|}{$\begin{array}{l}\rightarrow \text { aumenta de nivel. } \\
\uparrow \text { consigue el máximo nivel. }\end{array}$} \\
\hline
\end{tabular}

\section{Evaluación del CMD puesto en práctica}

\section{Evaluación de la docente y del diseño didáctico}

Se administró un cuestionario al final del Ciclo de mejora en el que se evaluaron distintas cuestiones respecto a la docente, los contenidos, actividades, metodología, ambiente y evaluación de las sesiones, mediante una escala con 5 niveles: muy alta, alta, media, baja y muy baja. Obteniéndose puntuaciones elevadas para las mismas. Se muestra la evaluación del profesorado, la metodología y los contenidos (Figuras 7, 8 y 9)

Jornadas de Formación e Innovación Docente del Profesorado | № 2 (2019)

Esta obra se distribuye con la licencia Creative Commons Reconocimiento-NoComercial-SinObraDerivada $\quad 4.0$ Internacional (CC BY-NC-ND 4.0.) 


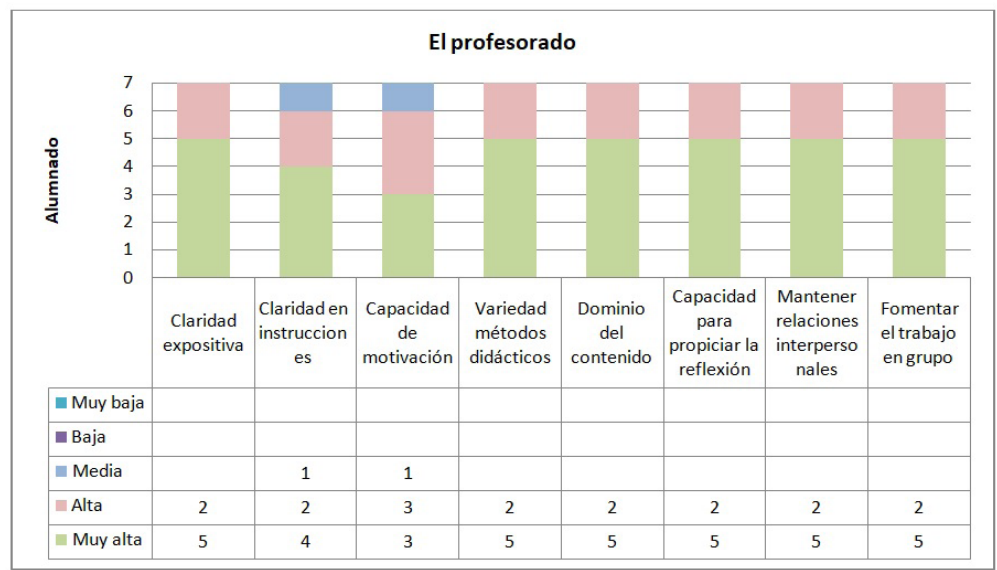

Figura 7. Evaluación del profesorado

\begin{tabular}{|c|c|c|c|c|c|c|}
\hline \multicolumn{7}{|c|}{ Metodología } \\
\hline \multirow{5}{*}{ 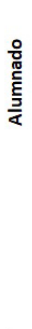 } & \multirow{5}{*}{$\begin{array}{l}7 \\
6 \\
5 \\
4 \\
3 \\
2 \\
1 \\
0\end{array}$} & & & & \\
\hline & & & & & & \\
\hline & & & & & & \\
\hline & & & & & . & \\
\hline & & $\begin{array}{c}\text { Presentación } \\
\text { de Teorías y } \\
\text { Conceptos }\end{array}$ & $\begin{array}{c}\text { Demostración } \\
\text { de la teoría o } \\
\text { destreza (en } \\
\text { vivo, en } \\
\text { vídeo, en } \\
\text { audio, por } \\
\text { escrito) }\end{array}$ & $\begin{array}{c}\text { Trabajosa } \\
\text { realizar fuera } \\
\text { del curso }\end{array}$ & $\begin{array}{l}\text { Presentación } \\
\text { de materiales } \\
\text { de aprendizaje }\end{array}$ & $\begin{array}{c}\text { Reflexión } \\
\text { sobre la } \\
\text { propia } \\
\text { práctica }\end{array}$ \\
\hline \multicolumn{7}{|c|}{ - Muy baja } \\
\hline \multicolumn{7}{|c|}{ - Baja } \\
\hline \multicolumn{7}{|c|}{ Media } \\
\hline & & 4 & 3 & 3 & 3 & 4 \\
\hline & alta & 3 & 3 & 3 & 3 & 3 \\
\hline
\end{tabular}

Figura 8. Evaluación de la metodología

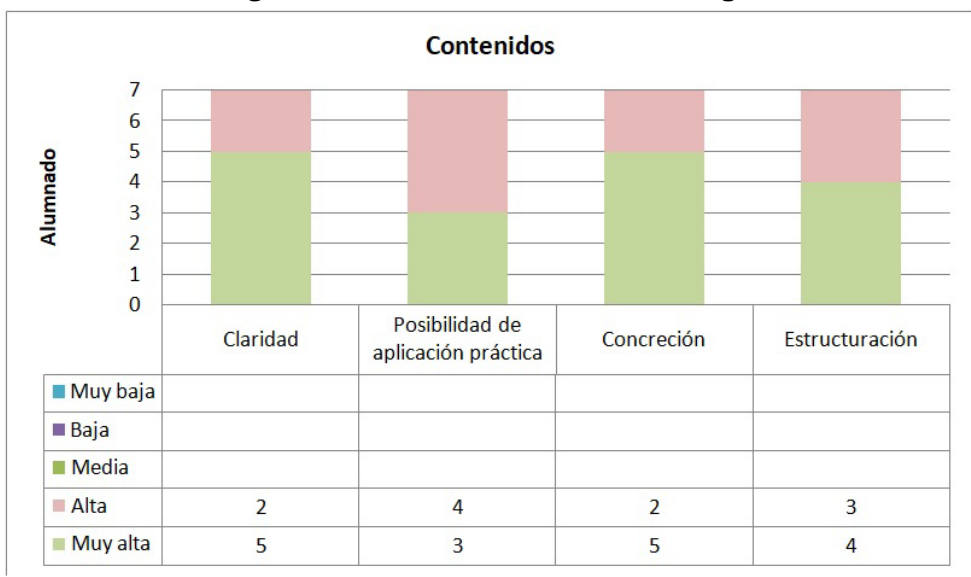

Figura 9. Evaluación de los contenidos

Jornadas de Formación e Innovación Docente del Profesorado | № 2 (2019) 


\section{Cuestiones a mantener y cambios a introducir en el CIMA}

Como cuestiones a mantener en la enseñanza de estas temáticas, me gustaría seguir realizando pequeños ciclos de mejora docente en los que la gamificación tenga especial relevancia en mis clases; utilizar diferentes metodologías en el aula como roles playing, videoforums o casos clínicos dentro de una misma sesión cuando es tan amplia, porque así la docencia es más dinámica; diseñar cuestionarios y evaluar sus respuestas para analizar los mapas mentales del alumnado.

Como cambios a introducir me planteo elaborar mapas de contenidos seleccionando los de aspectos más relevantes, ya que he podido analizar que la temporalización en algunos casos no se ha cumplido porque se ha invertido tiempo en otros contenidos de menos relevancia. También me gustaría introducir nuevas técnicas de motivación, no solo las distintas frases que he utilizado.

\section{Aspectos que se pretenden incorporar a la práctica docente habitual}

- Llevar un diario del profesor para reflexionar por escrito sobre la práctica docente.

- Seleccionar los contenidos más relevantes de implicación a su práctica futura.

- Elaborar cuestionarios iniciales y finales y analizar sus repuestas para construir las escaleras de aprendizaje.

- Reformular el modelo metodológico si no se adapta al alumnado.

Jornadas de Formación e Innovación Docente del Profesorado | № 2 (2019)

Esta obra se distribuye con la licencia Creative Commons 


\section{Principios didácticos que han guiado la experiencia}

- La dinámica del aula debe ser fruto de una negociación entre los estudiantes y yo misma, como docente.

- Mi metodología se basa en el abordaje de problemas para que los estudiantes construyan el conocimiento con mi orientación.

- Además de los contenidos conceptuales, son importantes los procedimentales y actitudinales en la formación de mis estudiantes.

- En mis clases trato de que los contenidos conecten con los problemas relevantes de la realidad.

- Debo evaluar los modelos mentales iniciales de los estudiantes e ir valorando su grado de aprendizaje.

- Debo evaluar todos los elementos implicados en mi docencia (docente, metodología, contenidos, actividades, ambiente, evaluación) para analizarlos, reflexionar sobre ellos y mejorarlos.

- La práctica docente es un proceso cambiante, que se adapta al alumnado y a las necesidades de su entorno.

Jornadas de Formación e Innovación Docente del Profesorado | № 2 (2019)

Esta obra se distribuye con la licencia Creative Commons 


\section{Referencias bibliográficas}

Bain, K. (2007). Lo que hacen los mejores profesores universitarios. Valencia: Publicaciones Universidad de Valencia. ISBN: 978-84-370-6669-1.

Finkel, D. (2008). Dar clases con la boca cerrada. Valencia: Publicaciones Universidad de Valencia. ISBN:978-84-370-7268-5.

Porlán, R. (Coord.) (2017). Enseñanza Universitaria. Cómo mejorarla. Madrid: Editorial Morata. ISBN:978-84-7112-860-7

Porlán, R. y Martín, J. (1991). El Diario del Profesor. Díada: Sevilla. https://profesorailianartiles.files.wordpress. com/2013/03/diario-del-maestro.pdf

Jornadas de Formación e Innovación Docente del Profesorado | № 2 (2019)

Esta obra se distribuye con la licencia Creative Commons Reconocimiento-NoComercial-SinObraDerivada Internacional (CC BY-NC-ND 4.0.) 